\title{
Research on Optimal Allocation of Water Resources Based on Large-Scale System Decomposition and Coordination Model
}

\author{
Zhuoran LUO ${ }^{\mathrm{a}, \mathrm{b}}$, Jiahong LIU ${ }^{\mathrm{a}, \mathrm{b}, \mathrm{c}, 1}$, Weiwei SHAO ${ }^{\mathrm{b}}$, Yongxiang ZHANG $^{\mathrm{a}}$ and Ruitao \\ JIA $^{\text {a }}$ \\ ${ }^{a}$ Faculty of Architecture, Civil and Transportation Engineering, Beijing University of \\ Technology, Beijing 100124, China \\ ${ }^{\mathrm{b}}$ State Key Laboratory of Simulation and Regulation of Water Cycle in River Basin, \\ China Institute of Water Resources and Hydropower Research, Beijing 100038, China \\ ${ }^{\mathrm{c}}$ School of Transportation and Civil Engineering \& Architecture, Foshan University, \\ Guangdong 528000, China
}

\begin{abstract}
The construction of water resources optimal allocation model is the premise and foundation of solving and evaluating the optimal allocation model of water resources. The allocation of water resources includes not only the simple allocation of water resources, but also the protection of water resources and the analysis of the relationship between water supply and demand. Aiming at the problem of water shortage in the receiving area of water diversion from Hanjiang River to Weihe River, the large-scale system decomposition and coordination algorithm is used to optimally allocate the water use departments of each district of the water diversion area from Han to Wei River in Shaanxi Province, and establish the water diversion project from Han to Wei River. Optimal allocation model of water resources in the water receiving area. The results show that: in the 2030 planning level, the water supply of key cities, Xixian new district, medium/small cities, and industrial parks were 153.57, 368.16, 632.04, and 208.68 million m3, respectively, and the corresponding water shortage rate was $2.8 \%, 5.6 \%, 8.4 \%$, $11.2 \%$. The water supply sequence has a lower water shortage rate than the previous one, and the water shortage rate of the domestic water sector in key cities is only $1.2 \%$. From the water shortage situation of various water departments in 2030 , it can basically meet the water shortage of water receiving objects and effectively improve the water shortage in water receiving areas.
\end{abstract}

Keywords. Decomposition and coordination method, optimized configuration, large scale system, water resources, hanjiang to weihe river project

\section{Introduction}

The allocation of water resources play an important role in water conservancy work [1]. On the one hand, it is a useful tool for decision-makers to make macro decisions and weigh the advantage and disadvantages in the process of water resource allocation; on the other hand, it is also the core work in the decision-making process, such as

\footnotetext{
${ }^{1}$ Jiahong Liu, State Key Laboratory of Simulation and Regulation of Water Cycle in River Basin, China Institute of Water Resources and Hydropower Research, Beijing 100038, China; E-mail: liujh@iwhr.com.
} 
analyzing the carrying capacity of water resource, allocating initial water rights, and formulating water diversion schemes with different objectives. The optimization goal of the water resource allocation model is to control groundwater overexploitation under the premise of meeting the demand of water resources for the benign and sustainable development of the regional economy and society, and gradually restore the groundwater level to the level of the 1990s [2]; Through the joint dispatching of surface water, groundwater and external water transfer, the utilization rate of water resources can be improved and unnecessary waste can be reduced; The rational allocation of industrial and agricultural water, domestic water and environmental water can be realized by controlling the total water shortage and pollutant discharge [3].

The total amount of water resources in the catchment area of Hanjiang to Weihe River is insufficient, the shortage of water resources, the national economic water occupying the ecological environment water, the lack of unified planning for the development and utilization of water resources, the serious pollution of water environment, the serious lag of waste water treatment measures, the severe flood control situation in the middle and lower reaches, which have seriously affected the sustainable development of economy and society in the water receiving area [4]. In general, the deterioration of the ecological environment in the receiving area is caused by the shortage and unreasonable use of water resources. The most effective way to solve this problem is to increase the amount of water resources and allocate the water resources reasonably [5]. The allocation of water resources is to obtain a water allocation plan that maximize the benefits under the restrictive conditions such as natural constraints, engineering constraints, user demand constraints, considering the comprehensive benefits of the economy, society and the ecological environment. For the allocation of water resources, the limitation of water resources determines that it is often unable to meet the corresponding requirements of social economic development and ecological environmental protection at the same time [6].

In this study, the principle of water resources optimization modeling is to fully consider the complex water cycle mechanism, groundwater over-exploitation, water supply and demand balance, ecological environment restoration and other factors in the study area, take regional economic benefits, social benefits and ecological environment efficiency as the goal, and use the principle of large-scale system decomposition and coordination to establish an optimal allocation model of water resources.

\section{Materials and Methods}

\subsection{Study Area}

As shown in figure 1, the water receiving area from Han to Wei River in Shaanxi Province is located in the Guanzhong area from Baoji Gorge to Tongguan [7]. It is bounded by the Jialing River, Hanjiang River, Danjiang River and Nanluo River in the south, Shaanxi-Gansu province boundary from west to east, Zhangjiashan River and Luohe River-shaped head in the north, with a total area of $35489 \mathrm{~km}^{2}$. The water receiving area is bounded by the main stream of Weihe River, which is divided into four water resources divisions: Baoji Gorge to the north bank of Xianyang, Xianyang to the north bank of Tongguan, Xianyang to the south bank of Tongguan, with an area of $11392 \mathrm{~km}^{2}, 6480 \mathrm{~km}^{2}, 9739 \mathrm{~km}^{2}$ and $7878 \mathrm{~km}^{2}$ respectively. It is an area with good 
economic foundation, superior natural conditions, profound humanities and history, and great development potential in the western region of China.

The Hanjiang to Weihe River Project refers to a water conservancy project that takes Han water from Yang County in Hanzhong City, Shaanxi Province, through the Qinling Mountains, into the Guanzhong area, and finally into the Weihe River. The project mainly consists of five parts: Huangjinxia Reservoir Hub, Huangjinxia Water Source Pumping Station, Huangjinxia-Sanhekou Water Transfer Project, Sanhekou Reservoir and Qinling Tunnel. After the project is completed, it can basically meet the water needs of 4 key cities, 5 new cities, 11 counties and 2 industrial parks, gradually return the occupied agricultural and ecological water, alleviate the contradiction between urban and rural, ecological water, and provide conditions for the allocation of water resources in Shaanxi Province.

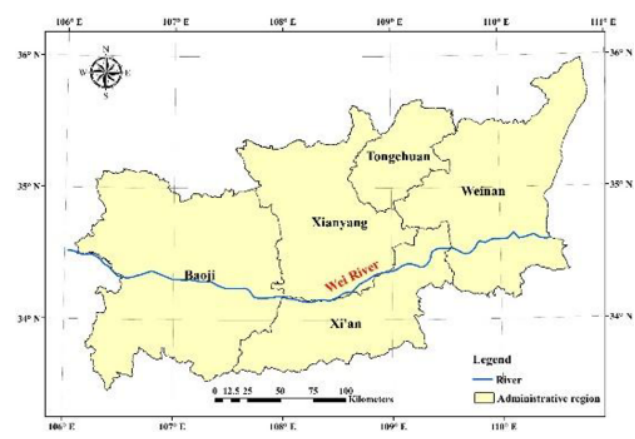

Figure 1. Schematic diagram of Hanjiang to Weihe River Project in Shaanxi Province.

\subsection{Solving Ideas of Decomposition and Coordination Method}

The optimization steps of decomposition and coordination method are generally as follows [8]: first, the mathematical model of the real system of the research object is established; Secondly, according to the nature and characteristics of the model and the principle of easy solution, the appropriate hierarchical structure is selected, and the large-scale system problem is transformed into a subsystem with a hierarchical relationship by decomposition. Thirdly, these subsystems (relatively independent) are respectively optimized according to their respective optimization conditions; Finally, the whole large-scale system is optimized through the coordination relationship among all levels. As far as the solver is concerned, decomposition is the structural design of large-scale system algorithm, and coordination layer is the guarantee of global optimization of large-scale system [9]. In view of the current situation of insufficient water supply in the water receiving area and the limited water transfer volume of the Hanjiang to Weihe River Project, after each city has allocated a certain amount of water, it will allocate a reasonable amount of water to the city's water sector, thus forming the optimal water distribution process of water resources that can maximize the benefits in the receiving area. The water distribution among different cities belongs to the optimization process of the whole water receiving area system, while the water distribution among different water departments in cities belongs to the optimization of each urban subsystem.

As shown in figure 2, the urban water sector (life, industry, agriculture, ecology) is regarded as the first layer, its function is to allocate the water quantity of the second 
and third layer (water quantity optimization between urban regions) model to the $i$-th city, and make the optimal allocation among the water use departments in the city [10]. The area where the water receiving area is located as the second layer, and the regional level where the water receiving area is located as the third layer. The second and third layers are used to solve the optimal allocation among urban areas in the water receiving area when the water is in short supply, and their function is to use the feedback information of the first layer to optimally allocate the public water supply among important cities. When the model runs, the third layer allocates a certain amount of water to the second layer and then to each subsystem of the first layer. Each subsystem is independently optimized after a certain amount of water is given, and the optimal allocation result is obtained, and the optimal value is fed back to the second layer and the third layer. According to the feedback information, the third layer calculates the optimal fitness of the whole system, and changes the last allocation of water to get a new set of water allocation results and the optimal fitness of the whole system until the optimal fitness of the whole system is obtained. The three layers are connected by the water supply allocated to each city to form a large-scale system with a three-tier pedigree structure, which is suitable for solving with a large-scale system decomposition and coordination model.

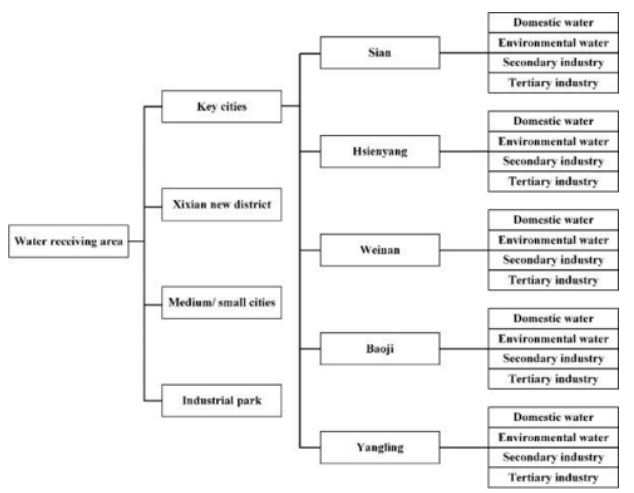

Figure 2. Water resources optimal allocation of water receiving area.

\section{Results and Discussion}

\subsection{Calculation results of Model Parameters}

\subsubsection{Determination of the Equity Coefficient B of Cities and their Water Sector}

Equity coefficient $\beta^{k}$ (k city level water supply order coefficient) means that in the process of water supply, due to the difference of city importance and water use evaluation index, the priority order of water supply in each water receiving area is artificially determined according to expert opinions, and then transformed into [0,1] interval. The order coefficients of urban water supply are: 0.4 for key cities, 0.3 for Xixian new district, 0.2 for medium/small cities and 0.1 for Yangling demonstration area. In the same way, according to the principle of fairness, urban domestic water should be satisfied first, then urban ecological water should be allocated scientifically, and finally it should be allocated to production water. Similarly, the fairness 
coefficients of water departments in the city are calculated by using the formula of water supply sequence coefficient in the water receiving area: 0.4 for domestic water, 0.3 for environmental water, 0.2 for secondary production, and 0.1 for tertiary production.

\subsubsection{Determination of Target Weight Coefficient $\lambda$}

Many methods for solving multi-objectives have been introduced in the previous section. Here, the method of turning more into less is adopted, that is, turning multiple objectives into a single objective, and using linear weighted sum method, the weight coefficient of economic objective (maximum net benefit of water supply) is 0.5 . The weight coefficient of the social target (minimum total water shortage in water receiving area) is 0.3 , and the weight coefficient of environmental target (minimum sewage discharge) is 0.2 .

\subsubsection{Benefits, Costs and Sewage Discharge Coefficient}

The industrial benefit coefficient is determined by the method of sharing the total industrial output value: the benefits of the tertiary industry, living and urban environmental water are complex and difficult to determine. They have not only economic factors but also social factors. According to the principle of giving priority to life and environment in this paper, they are given a larger value.

When the water source is surface water, the water price is taken as its cost coefficient; When the water source is groundwater, the sum of water resources fee and water delivery cost is taken as its cost coefficient; In other cases, the sewage treatment fee is taken as its cost coefficient.

Sewage discharge coefficient is the ratio of sewage discharge and water consumption in a certain calculation time. According to the living standard of urban planning, the perfection of water supply and drainage facilities, and the urban drainage facilities planning penetration rate, combined with the proportion of the tertiary industry output value in the GDP. According to the city's industrial structure and production equipment, the degree of technological advancement and the penetration rate of urban drainage facilities determine the secondary industry wastewater discharge coefficient.

\subsection{Optimal Allocation of Water Resources}

Based on the water supply planning and the present water resources development and utilization degree of the Hanjiang to Weihe River Project in Shaanxi Province, according to the objective functions and constraints listed above, the water resources are allocated for the planning level of the water receiving area, and the results of optimal allocation of water resources in the water-receiving area in 2030 are obtained.

It can be seen from table 1 that in the planning level year of 2030, the water supply of key cities, Xixian new area, medium/small cities and industrial parks are 1535.76, $368.16,632.04$ and 208.68 million $\mathrm{m}^{3}$ respectively, and the corresponding water shortage rates are $2.8 \%, 5.6 \%, 8.4 \%$ and $11.2 \%$. Similarly, the water demand, water supply and water shortage rate of each water department can also be obtained from table 2. The concept of fairness coefficient is used to make the water receiving areas at the same level have the same water shortage rate, and the water shortage rate of the water supply order is less than that of the previous one. The water shortage rate of the 
domestic water sector in key cities is only $1.2 \%$. According to the water shortage in water use departments in 2030, the decomposition and coordination method distributes water equally among the living, ecological, secondary and tertiary industries, and maximizes the target function value.

Table 1. Optimization results of water resources allocation at the regional level (million $\mathrm{m}^{3}$ ).

\begin{tabular}{llllll}
\hline Water receiving object & Demand & Supply & Transfer water & Total supply & Shortage \\
\hline Key cities & 1580 & 1064 & 471.93 & 1535.76 & $2.80 \%$ \\
Xixian new district & 390 & 198 & 169.99 & 368.16 & $5.60 \%$ \\
Medium/small cities & 690 & 264 & 366.37 & 632.04 & $8.40 \%$ \\
Industrial park & 235 & 19 & 189.4 & 208.68 & $11.20 \%$ \\
Total & 2895 & 1545 & 1197.69 & 2744.64 & $5.20 \%$ \\
\hline
\end{tabular}

Table 2. Optimization results of water resources allocation in water departments (million $\left.\mathrm{m}^{3}\right)$.

\begin{tabular}{llllll}
\hline $\begin{array}{l}\text { Water receiving } \\
\text { object }\end{array}$ & Index & $\begin{array}{l}\text { Domestic } \\
\text { water }\end{array}$ & $\begin{array}{l}\text { Environmental } \\
\text { water }\end{array}$ & $\begin{array}{l}\text { Secondary } \\
\text { industry }\end{array}$ & $\begin{array}{l}\text { Tertiary } \\
\text { industry }\end{array}$ \\
\hline \multirow{3}{*}{ Key cities } & Demand & 490.41 & 118.53 & 807.7 & 163.56 \\
& Supply & 484.7 & 115.77 & 779.51 & 155.95 \\
& Shortage & $1.2 \%$ & $2.3 \%$ & $3.5 \%$ & $4.7 \%$ \\
Xixian new district & Demand & 124.54 & 32.07 & 172.67 & 60.54 \\
& Supply & 121.67 & 30.59 & 160.75 & 54.97 \\
\multirow{3}{*}{$\begin{array}{l}\text { Medium/ies } \\
\text { Shortage }\end{array}$} & $2.3 \%$ & $4.6 \%$ & $6.9 \%$ & $9.2 \%$ \\
& Demand & 160.51 & 36.08 & 441.15 & 50.44 \\
Industrial park & Supply & 155.23 & 33.71 & 397.63 & 43.8 \\
& Shortage & $3.3 \%$ & $6.6 \%$ & $9.9 \%$ & $13.2 \%$ \\
& Demand & 65.8 & 13.51 & 131.02 & 24.35 \\
& Supply & 62.84 & 12.29 & 113.31 & 19.96 \\
\hline
\end{tabular}

It can be seen from figure 3 that the proportion of surface water supply is the largest in key urban areas, reaching 36.5\%. The Hanjiang to Weihe River Project has carried out a certain proportion of water transfer at all regional levels, and the largest proportion of water transfer to industrial parks is $80.7 \%$. According to the proportion of water shortage, each water receiving object has some water shortage in different degrees, but compared with before the Hanjiang to Weihe River, the water shortage situation has been obviously improved. When the amount of water diverted from Hanjiang to Weihe River is distributed according to the demand, the objects with the earlier water supply order can basically achieve the balance of supply and demand, and the water supply objects with the lower water supply order have also greatly improved their water shortage. The water shortage in the water receiving area is 152.31 million $\mathrm{m}^{3}$, of which the water shortage rate in key cities is only $2.8 \%$. This shows that the 1.5 billion $\mathrm{m} 3$ of water transferred from Hanjiang to Weihe River (converted to 1.2 billion $\mathrm{m}^{3}$ in the receiving area), which basically meets the water shortage of the receiving object. It can effectively improve the shortage of water resources in the water receiving area. 


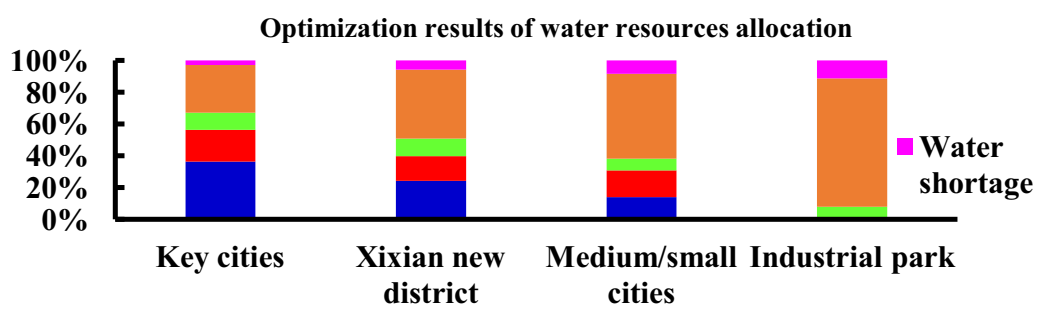

Figure 3. Optimized statistics on the allocation ratio of each water source.

\section{Conclusions}

In this study, the general idea, solution method, objective function and constraint conditions of the large-scale system decomposition and coordination model are analyzed, and the objectives, principles, classification and basic modes of optimal allocation of water resources are discussed, and the optimization process of the model is analyzed. Under the principles of efficiency, safety, coordination and fairness, a water resources allocation model is constructed, which aims at maximizing the comprehensive benefits of social economy and ecological environment, and takes the water supply, water demand and water supply capacity as constraints. Solving the problem of joint dispatch and regulation of the water diversion from the Hanjiang to Weihe River and the local water sources in Guanzhong will also play an important role in the Guanzhong water supply system to be constructed. It is of far-reaching significance to the economic operation of the Hanjiang to Weihe River Project and to ensure the safety of the water supply in Guanzhong.

\section{Acknowledgments}

This work was supported by the National Key Research and Development Program of China (Grant No. 2018YFC1508203, 2016YFC0401401), the Natural Science Basic Research Program of Shannxi Province (2019JLZ-15), the Chinese National Natural Science Foundation (No. 51739011) and the Research Fund of the State Key Laboratory of Simulation and Regulation of Water Cycle in River Basin (Grant No. SKL2020ZY03).

\section{References}

[1] Delorit JD, Block PJ. Promoting competitive water resource use efficiency at the water-market scale: an intercooperative demand equilibrium-based approach to water trading. Water Resources Research. 2018 Aug; 54(8): 5394-5421.

[2] Massuel S, Riaux J. Groundwater overexploitation: why is the red flag waved? Case study on the Kairouan plain aquifer (Central Tunisia). Hydrogeology Journal. 2017 Sep; 25(6): 1607-1620.

[3] Xiong TY, He J, Pi X, Jiang S, Han F, Zheng Y. Joint operation of surface water and groundwater reservoirs to address water conflicts in arid regions: an integrated modeling study. Water. 2018 Aug; 10(8): 1105.

[4] Liu J, Yan F, Niu C, Xu H, Qin T, Yang H. Key issues on water distribution system of the water transfer project from Hanjiang River to Weihe River. Journal of China Institute of Water Resources and Hydropower Research. 2014 Sep; 12(03): 244-248+257. 
[5] Liu X, Guo P, Tan Q, Zhang F, Huang Y, Wang Y. Drought disaster risk management based on optimal allocation of water resources. Natural Hazards. 2021 Mar; 108:285-308.

[6] Saeid N, Omid BH, Mostafa K, Xuefeng C. Multi-objective optimization for allocation of surface water and groundwater resources. Science of the Total Environment. 2021 Mar; 776(12): 146026.

[7] Shao W, Liu X, Xu H, Niu C, Zhou Z, Huang H. Study on the water supply and water use processes in the benefited areas in water diversion from Han River to Wei River Project. Journal of Water Resources Research. 2013 Jun; 2(5): 331-336.

[8] Dong X, Xu L, Xu Z. Research on the optimal allocation of water resources in Xingjiadu irrigation area based on large-scale system decomposition-coordination method. Yellow River. 2021 Jan; 43(04): 8288.

[9] Luo F, Yang W, Zhang T, Wei G, Liu Y. Research on decomposition and coordination planning method for information-side and physical-side of power distribution system. Journal of Residuals Science \& Technology. 2016 Jun; 13(8).

[10] Wang X, Tang Q. Study on large-scale system decomposition and coordination method for optimal operation of Qingjiang cascade hydropower stations. Water science and Engineering Technology. 2017 Oct; 2017(05): 78-82. 